\title{
The TanDEM-X Mission: Overview and Interferometric Performance
}

\author{
Sigurd Huber ${ }^{\# 1}$, Gerhard Krieger \#2 \\ \# Institut für Hochfrequenztechnik und Radarsysteme, Deutsches Zentrum für Luft- und Raumfahrt e.V. (DLR) \\ Münchner Straße 20, D-82234 Weßling \\ ${ }^{1}$ sigurd.huberedlr.de \\ 2gerhard.kriegeredlr. de
}

\begin{abstract}
TanDEM-X (TerraSAR-X add-on for Digital Elevation Measurement) is an innovative spaceborne $X$-band earth observation mission that will be launched in October 2009. This paper gives an overview of the TanDEM-X mission concept, summarizes the basic products and illustrates the achievable performance. In more detail the effect of quantization on the interferometric performance is discussed. Finally new imaging techniques are outlined.
\end{abstract}

\section{INTRODUCTION}

The primary objective of the TanDEM-X mission is the generation of a world-wide, consistent, timely, and high precision digital elevation model aligned with the HRTI-3 specification as the basis for a wide range of scientific research, as well as for commercial DEM production [1]. This goal will be achieved by means of a second, TerraSAR-X like satellite flying in close orbit configuration with TerraSAR-X. Both satellites will then act as a large single-pass SAR interferometer with the opportunity for flexible baseline selection. This enables the acquisition of highly accurate cross-track and along-track interferograms without the inherent accuracy limitations imposed by repeat-pass interferometry due to temporal decorrelation and atmospheric disturbances. Besides the primary goal of the mission, several secondary mission objectives based on along-track interferometry as well as new techniques with bistatic SAR have been defined which represent an important and innovative asset of the mission. TanDEM-X will be implemented in the framework of a publicprivate partnership between the German Aerospace Center (DLR) and EADS Astrium GmbH, as for TerraSAR-X. The launch of TanDEM-X is planned for October 2009.

\section{MISSION CONCEPT}

The TanDEM-X mission is an extension of the TerraSAR$\mathrm{X}$ mission, co-flying a second satellite of nearly identical capability in a close formation. Among several options investigated, the HELIX formation [2] finally has been selected for operational DEM generation. The TerraSAR-X satellite (TSX), as basis for TanDEM-X, is not only a high performance SAR system with respect to SAR image and operational features, but it has already built in all necessary features required for the implementation of the TanDEM-X mission. Examples are additional X-band horn antennas for inter-satellite phase synchronization, the availability of a dual-frequency GPS receiver for precise orbit determination, excellent RF phase stability of the SAR instrument, and pulse repetition frequency $(P R F)$ synchronization based on GPS as a common time reference. The second satellite (TDX) will be as much as possible a rebuild of TSX with only minor modifications like an additional cold gas propulsion system for formation fine tuning and an additional S-band receiver to enable the reception of status and GPS position information broadcasted by TSX. This guarantees a low development risk and it offers the possibility for a flexible share of operational functions among the two satellites. The TDX satellite will be designed for a nominal lifetime of $5 \frac{1}{2}$ years and has a nominal overlap with TSX of 3 years. Note in this context that TSX holds consumables and resources for up to seven years of operation, allowing for a potential prolongation of the overlap and the TanDEM-X mission duration. The instruments on both satellites are advanced high resolution X-band synthetic aperture radars based on active phased array technology, which can be operated in Spotlight, Stripmap, and ScanSAR mode with full polarization capability [3]. The center frequency of the instruments is $9.65 \mathrm{GHz}$ with a selectable SAR chirp bandwidth of up to $300 \mathrm{MHz}$. The active phased array antenna, which has an overall aperture size of $4.8 \mathrm{~m} \times 0.7 \mathrm{~m}$, is fixed mounted to the spacecraft body and incorporates 12 panels with 32 dual-pol slotted waveguide sub-arrays each. This enables agile beam pointing and flexible beam shaping.

\section{A. Interferometric Data Acquisition}

Interferometric data acquisition with the TanDEM-X satellite formation can be achieved in different operational modes: Examples are Bistatic, Monostatic, and Alternating Bistatic operation. The three interferometric configurations may further be combined with different TSX and TDX SAR imaging modes like Stripmap, ScanSAR, Spotlight, and Sliding Spotlight. Operational DEM generation is planned to be performed using the bistatic InSAR Stripmap mode. This mode uses either TSX or TDX as a transmitter to illuminate a common radar footprint on the Earth's surface. The scattered signal is then recorded by both satellites simultaneously. This simultaneous data acquisition makes dual use of the available transmit power and is mandatory to avoid possible errors from temporal decorrelation and atmospheric disturbances. 


\section{B. Relative Phase Referencing}

A peculiarity of the bistatic data acquisition is the use of independent oscillators for modulation and demodulation of the radar pulses. Any deviation between the two oscillators will hence cause a residual modulation of the recorded azimuth signal. The impact of oscillator phase noise in bistatic SAR has been analyzed in [4] where it is shown that oscillator noise may cause significant errors in both the interferometric phase and SAR focusing. The stringent requirements for interferometric phase stability in the bistatic mode will hence require an appropriate relative phase referencing between the two SAR instruments or an operation in the alternating bistatic mode. For TanDEM-X, a dedicated inter-satellite X-band synchronization link will be established by a mutual exchange of radar pulses between the two satellites. For this, the nominal bistatic SAR data acquisition is shortly interrupted, and a radar pulse is redirected from the main SAR antenna to one of six dedicated synchronization horn antennas mounted on each spacecraft. The pulse is then recorded by the other satellite which in turn transmits a short synchronization pulse. By this, a bidirectional link between the two radar instruments will be established, which allows for mutual phase referencing without exact knowledge of the actual distance between the satellites. On ground, a correction signal can then be derived from the recorded synchronization pulses which compensates the oscillator induced phase errors in the bistatic SAR signal. The performance of such a synchronization link has been investigated in [5]. Fig. 1 shows the predicted standard deviation

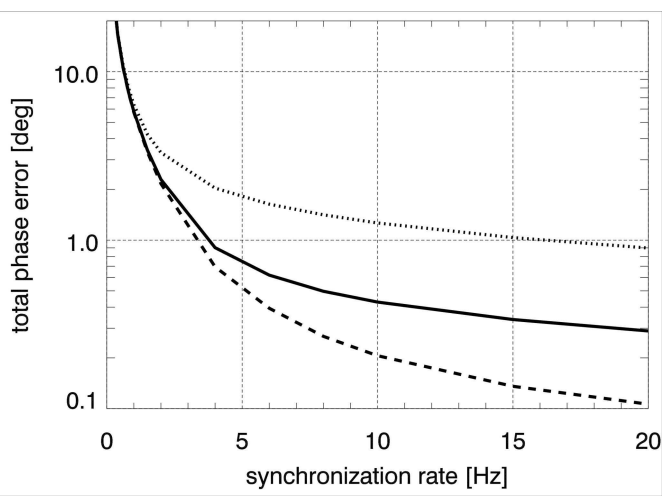

Fig. 1. Standard deviation of the total synchronization link phase error as a function of the synchronization frequency. The three curves are for signalto-noise ratios of $20 \mathrm{~dB}$ (dotted), $30 \mathrm{~dB}$ (solid), and $40 \mathrm{~dB}$ (dashed). The standard deviation of the total phase error should be lower than $1^{\circ}$ to meet the TanDEM-X requirements

of the residual phase errors after synchronization as a function of the update frequency of the synchronization signals for different signal-to-noise ratios $(S N R)$ of the bidirectional link. The actual $S N R$ varies with the distance between the satellites as well as their relative attitude. For the typical DEM data acquisition mode with baselines below $1 \mathrm{~km}$, the $S N R$ will be in the order of $30 \mathrm{~dB}$ to $40 \mathrm{~dB}$, and it becomes clear that a phase error below $1^{\circ}$ can be achieved for synchronization frequencies of $5 \mathrm{~Hz}$.

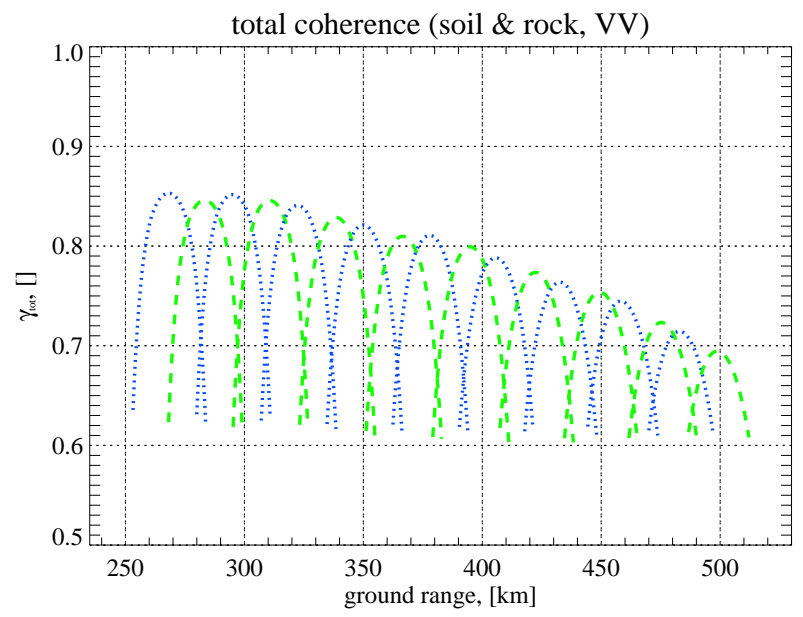

Fig. 2. Total coherence for the two interferometric acquisitions showing a clear decay towards far range

\section{Performance AnAlysis}

This section investigates the interferometric performance of TanDEM-X. For this, an interferometric data acquisition in bistatic Stripmap mode will be assumed. Major factors which affect the relative height accuracy are the radiometric sensitivity of each SAR instrument, range and azimuth ambiguities, quantization noise, processing and co-registration errors as well as surface and volume decorrelation, scaled by the baseline length [2]. The key quantity in estimating the interferometric performance is the coherence which has been computed by the product

$$
\gamma_{\text {tot }}=\gamma_{S N R} \gamma_{\text {quant }} \gamma_{\mathrm{amb}} \gamma_{\text {coreg }} \gamma_{\text {geo }} \gamma_{\mathrm{az}} \gamma_{\mathrm{vol}} \gamma_{\mathrm{temp}}
$$

where the right hand side describes the different error contributions due to the limited $S N R\left(\gamma_{S N R}\right)$, quantization $\left(\gamma_{\text {quant }}\right)$, ambiguities $\left(\gamma_{\mathrm{amb}}\right)$, limited coregistration accuracy $\left(\gamma_{\text {coreg }}\right)$, baseline decorrelation $\left(\gamma_{\text {geo }}\right)$, relative shift of Doppler spectra $\left(\gamma_{\mathrm{az}}\right)$, volume decorrelation $\left(\gamma_{\mathrm{vol}}\right)$, and temporal decorrelation $\left(\gamma_{\text {temp }}\right)$. The coherence for soil and rock surfaces is presented in Fig. 2. Depending on the number of looks the coherence results in an interferometric phase error $\Delta \phi$, which transforms into the height error $\Delta h$ proportional to the height of ambiguity $h_{\mathrm{amb}}$.

$$
\Delta h=h_{\mathrm{amb}} \frac{\Delta \phi}{2 \pi} .
$$

Fig. 3 shows the result of the interferometric performance analysis for two different ambiguous heights corresponding to different baseline lengths in the order of $300 \mathrm{~m}$ and $400 \mathrm{~m}$, respectively.

\section{A. Performance in High Dynamic Backscatter Terrain}

In the afore presented performance prediction, a homogeneous backscatter distribution was assumed. This is an assumption which holds for example for large vegetated areas like rain forest or for deserts. But in densely populated areas, a high dynamic range in backscatter can be expected. Especially 


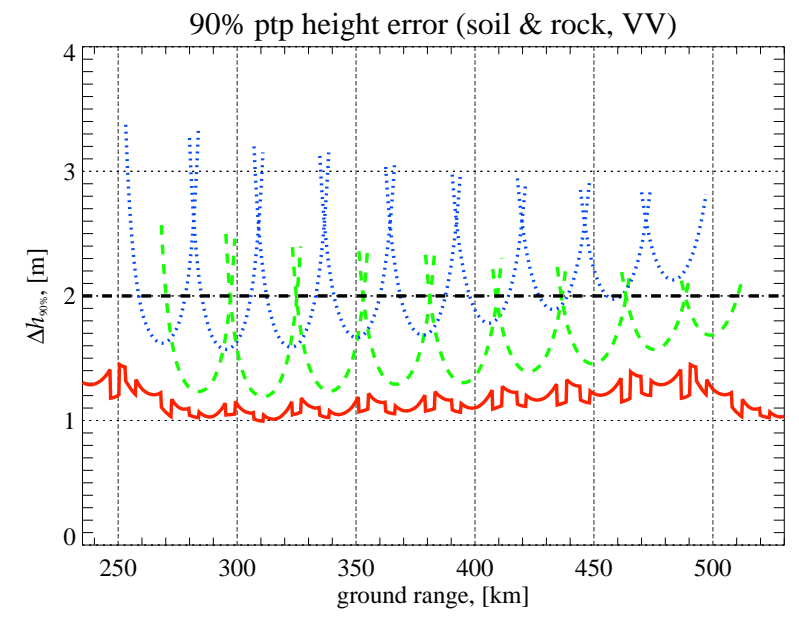

Fig. 3. Height accuracy for a height of ambiguity of $40 \mathrm{~m}$ (dotted) and $30 \mathrm{~m}$ (dashed). The lower solid curve shows the error resulting from the combination of multiple swaths. All errors are point-to-point height errors for a $90 \%$ confidence interval

man made structures like urban areas will reflect back more energy than natural scenes. Of special importance are land city transitions, where low and high scatterers are in close neighborhood.

Generally, a quantized signal is degraded by two error sources - granular noise and overload distortion or clipping errors. The effect considered here is called low scatterer suppression and is caused by inhomogeneous backscatter distributions. In the following the impact of quantization of signal returns from heterogeneous backscatter scenes on the height accuracy shall be analysed.

1) The Quantization Effect: The quantizer used in the TanDEM-X and TerraSAR-X satellite, respectively, is an 8 Bit Analog-to-Digital Converter (ADC) followed by a Block Adaptive Quantizer (BAQ). The BAQ quantizes blocks of 128 samples of the input raw data stream $u$ separately for the inphase- and quadrature-channel $\left(u_{\mathrm{I}}\right.$ and $\left.u_{\mathrm{Q}}\right)$. Based on the signal statistics, BAQ specific parameters are calculated, which can vary from block to block. The compression levels are $8: 2,8: 3,8: 4,8: 6$ and BAQ8:8, the last corresponds to BAQ bypass. Fig. 4 shows on the left side the inphase-channel
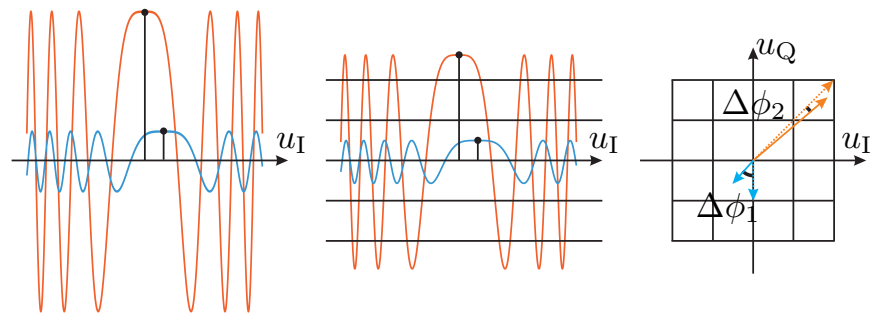

Fig. 4. Quantization of SAR raw data

$u_{\text {I }}$ consisting of two chirp signals originating from two point sources in close vicinity. This signal is weighted in order to fit in a fixed amplitude interval and quantized by means of the
ADC and the BAQ as indicated in the middle image. The low magnitude signal will be quantized very roughly and therefore be distorted by more granular noise than the strong signal. The resulting interferometric phase errors in the right image are denoted with $\Delta \phi_{1}$ and $\Delta \phi_{2}$, respectively. In the worst case, the phase error is $45^{\circ}$.

After focusing, in the SAR images both, the strong and the weak scatterers, are resolved. Consequently, regions with strong scatterers, which are quantized adequately will show low phase errors, while low backscatter regions are formidably more distorted. The amount of granular noise depends on the dynamic range of the backscatter.

2) Simulations: In a first approach an artificial scene with a rectangular scatterer profile in azimuth direction was simulated. The backscatter step height $\Delta \sigma_{0}$ is $15 \mathrm{~dB}$, which can be regarded as typical mean dynamic range in urban areas for X-band. Fig. 5 shows the azimuth scatterer profile and the
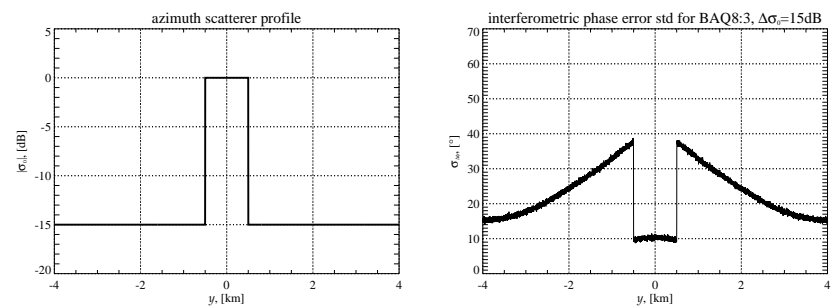

Fig. 5. Azimuth scatterer profile (left) and standard deviation of the interferometric phase error (right)

corresponding standard deviation of the interferometric phase error for a BAQ compression ratio of 8:3. The increase of the phase errors towards the region with high scatterer power between $\pm 500 \mathrm{~m}$ can be clearly observed. The extension of the zone with increased errors is proportional to the azimuth beam width, which is in the order of $4 \mathrm{~km}$ for an incidence angle of $30^{\circ}$. In range direction the errors are spread proportional to the transmitted pulse length.

The following simulations are based on TerraSAR-X measurement data over Cairo, Egypt. This scene offers a high dynamic range in backscatter power. To make the low scat-
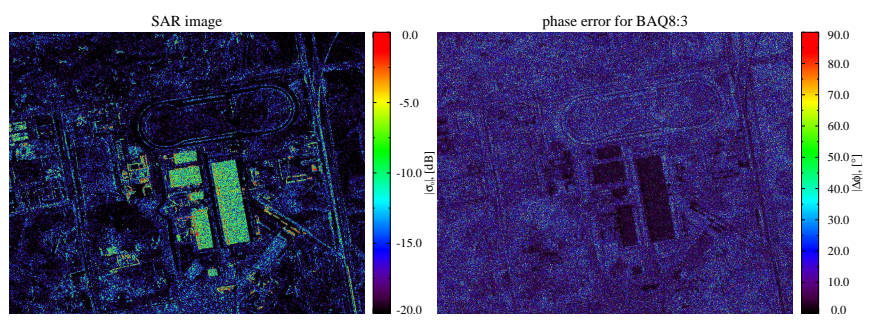

Fig. 6. SAR image with high dynamic range (left) and corresponding interferometric phase error for BAQ8:3 (right)

terer suppression effect visible, a small area including some buildings was analysed. The left plot in Fig. 6 shows the SAR image with a mean backscatter dynamic in the order of $15 \mathrm{~dB}$ to $20 \mathrm{~dB}$. The corresponding phase error is depicted on the right side. As expected, the strong scatterers are less distorted than the low scatterer regions. The histogram in Fig. 

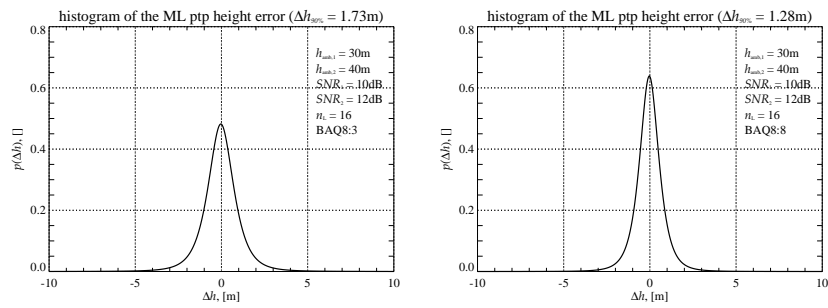

Fig. 7. Histogram for the point-to-point height error after optimum combination of the two interferometric acquisitions for BAQ8:3 (left) and BAQ8:8 (right)

7 shows the distribution of the height errors for BAQ8:3 (left) in comparison to BAQ8:8. This simulation is based on a large part of the Cairo scene. Here a mean $S N R$ of $10 \mathrm{~dB}$ and $12 \mathrm{~dB}$ for the two interferometric acquisitions is assumed. These are typical values observed in TerraSAR-X stripmap images. The $90 \%$ height error increases from $1.28 \mathrm{~m}$ to $1.73 \mathrm{~m}$.

\section{New Imaging TeChniques}

The TanDEM-X mission will provide the remote sensing scientific community with a unique data set to exploit the capability of new bistatic radar techniques and to apply these innovative techniques for enhanced geo- and biophysical parameter retrieval. TanDEM-X is predestined for along-track interferometry (ATI), which compares the phase of two complex SAR images acquired in identical geometries but separated by a short time interval. Digital Beamforming combines the RF signals from a set of small non-directional antennas to simulate a large directional antenna. Due to the split antennas and dual receiver channels of TSX and TDX, four phase centers can be obtained in a tandem mode.

\section{A. Very Large Baseline Cross-Track Interferometry}

Very large baseline interferometry takes advantage of the high RF bandwidth of the TSX and TDX satellites, which allows for coherent data acquisitions with baselines of up to $5 \mathrm{~km}$ and more. Note that less than $5 \%$ of the maximum possible (critical) baseline length is used during nominal DEM data acquisition. Large baseline interferograms can hence significantly improve the height accuracy, but the associated low height of ambiguity requires a combination of multiple interferograms with different baseline lengths to resolve phase ambiguities. By this, it becomes possible to derive DEMs with HRTI-4 like accuracy on a local or even regional scale. Fig. 8 shows a height accuracy example using the combination of four interferometric acquisitions with two additional orthogonal baselines in the order of $1.4 \mathrm{~km}$. From this example it becomes clear that in vegetation free and temporal stable areas DEMs with accuracies fulfilling the HRTI-4 requirements can be generated.

\section{CONCLUSION}

TanDEM-X is a highly innovative bistatic single-pass SAR interferometer which allows for the acquisition of unique remote sensing data products. The achievable height accuracy for global DEM generation with TanDEM-X is mainly limited

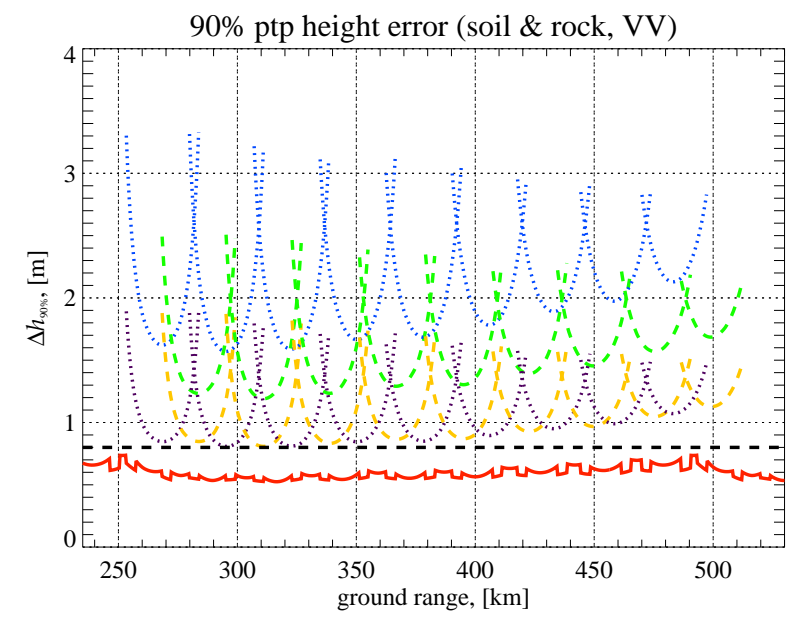

Fig. 8. Height accuracy prediction for four interferometric acquisitions with heights of ambiguities of $30 \mathrm{~m}$ and $40 \mathrm{~m}$ and two times $9 \mathrm{~m}$. The posting is $6 \mathrm{~m}$.

by the height of ambiguity that can finally be processed during phase unwrapping. The standard HRTI-3 DEM is a reasonable compromise between performance, processing, and data acquisition effort. A mission scenario has been developed which enables the acquisition of a global HRTI-3 DEM within less than three years. This concept includes several data takes with different baselines, different incident angles, and data takes from ascending and descending orbits to deal with difficult terrain like mountains, valleys, tall vegetation, etc. The TanDEM-X mission concept allocates also sufficient acquisition time and satellite resources to secondary mission goals like along-track interferometry or the demonstration of new bistatic radar techniques.

\section{ACKNOWLEDGEMENT}

The TanDEM-X project is partly funded by the German Federal Ministry of Economics and Technology (Förderkennzeichen 50 EE 0601).

\section{REFERENCES}

[1] G. Krieger, A. Moreira, D. Hounam, M. Werner, S. Riegger, and E. Settelmeyer, "A Tandem TerraSAR-X Configuration for Single-Pass SAR Interferometry," Proceedings of the International Conference on Radar Systems, 2004.

[2] G. Krieger, A. Moreira, H. Fiedler, I. Hajnsek, M. Werner, M. Younis, and M. Zink, "TanDEM-X: A Satellite Formation for High Resolution SAR Interferometry," IEEE Transactions on Geoscience and Remote Sensing, vol. 45, no. 11, pp. 3317-3341, 2007.

[3] M. Stangl, R. Werninghaus, B. Schweizer, C. Fischer, M. Brandfass, J. Mittermayer, and H. Breit, "TerraSAR-X technologies and first results," IEE Proc. - Radar, Sonar and Navigation, vol. 153, pp. 86-95, 2006.

[4] G. Krieger and M. Younis, "Impact of Oscillator Noise in Bistatic and Multistatic SAR," IEEE Geoscience and Remote Sensing Letters, vol. 3, pp. 424-428, 2006.

[5] M. Younis, R. Metzig, and G. Krieger, "Performance Prediction of a Phase Synchronization Link for Bistatic SAR," IEEE Geoscience and Remote Sensing Letters, vol. 3, pp. 429-433, 2006. 